\title{
Editorial
}

\section{Pharmacotherapy of post-traumatic stress disorder: going beyond the guidelines}

\author{
Jonathan Davidson
}

\section{Summary}

This article discusses the study of Harpaz-Rotem and associates, who examined patterns of medication use in first-diagnosed veterans with post-traumatic stress disorder (PTSD). It considers the difference between practice guidelines and actual prescribing; selectively identifies issues with antidepressants, antipsychotics, benzodiazepines and prazosin; and reviews the possible impact of new medications in the pipeline.

\section{Declaration of interests}

In the past 36 months, J.D. has received compensation for consulting with Edgemont, Turing and Tonix Pharmaceuticals; royalties in connection with publications by Springer, Guilford and McFarland Publishers and use of the Connor-Davidson Resilience Scale, Davidson Trauma Scale, Social Phobia Inventory (SPIN) and Mini-SPIN; service on the INTRUST Data Safety and Monitoring Board, University of California, San Diego.

\section{Copyright and usage}

(C) The Royal College of Psychiatrists 2016. This is an open access article distributed under the terms of the Creative Commons Non-Commercial, No Derivatives (CC BY-NC-ND) license.

Each drug group will be considered in turn, the matter of For some decades, there has been steady progress in the treatment of post-traumatic stress reactions. The growth of evidence-based medicine over the past 20 years has placed a premium on randomised controlled trials (RCTs), which are typically based on samples that do not represent real-world clinical practice. To offset this limitation, the field needs pragmatic or effectiveness studies in samples that represent more closely the types of patient seen in daily practice. For pharmacotherapy of post-traumatic stress disorder (PTSD), most guidelines agree that serotonin reuptake inhibitor (SRI) antidepressants are the drugs of first choice, being backed by a large body of evidence from RCTs. Their effects are modest but meaningful, and in line with the magnitude of gain for other conditions and not so different from the effects of psychotherapy in PTSD after allowing for study design differences. Many questions remain unanswered in the matter of drug therapy for PTSD, one of which concerns how prescribers actually use drugs and the similarity of use patterns to published guidelines. This account will expand on certain aspects of the publication by Harpaz-Rotem et al, ${ }^{1}$ which addressed the prescribing patterns of clinicians in the US Veteran's Health Administration (VHA or VA) system to treat initially diagnosed PTSD. It will consider their findings in the light of the evidence base for drug efficacy and how VHA guidelines have been applying this evidence.

Based on the evidence borrowed from non-veteran populations, current VA/Department of Defense guidelines recommend SRI antidepressants as first-choice drug therapy. ${ }^{2}$ Although two studies outside the United States have demonstrated benefit of fluoxetine and sertraline in veterans of military combat, ${ }^{3,4}$ almost all placebo-controlled trials of SRI drugs in US veterans with PTSD have been uniformly negative, conspicuous for the lack of even a trend towards the drug. ${ }^{5}$ A cynic might even wonder whether the guidelines are in effect recommending the use of a placebo which has more side-effects than a sugar pill. Serial negative results with SRI drugs suggest a need to revise the VA/ Department of Defense guidelines and to increase the comfort of military or VA physicians in broadening their treatment selections beyond currently favoured first-line recommended treatments, as has recently been advocated for psychotherapy because of residual morbidity and the demonstrated benefit of other therapies not considered first line. ${ }^{6,7}$ Indeed, one of the salutary lessons of the Harpaz-Rotem report is that prescribers of drug therapy do exactly that. polypharmacy will be discussed, and concluding remarks will sketch out five ways in which pharmacotherapy advances might impact the treatment of PTSD.

\section{Antidepressants}

Harpaz-Rotem et al studied the use of four drug classes in firstepisode PTSD among veterans of Iraq/Afghanistan, relating use patterns to comorbidity and five empirically derived symptom clusters. They also examined time between diagnosis and initiation of prescription. Their approach thus informs on how and when drugs are being prescribed in this population, although not whether they are effective. Their findings can be compared against VA practice directives which put simply (with some qualification), direct clinicians to choose SRI antidepressants as first-line pharmacotherapy in PTSD and discourage the use of benzodiazepines, as well as minimise the use of antipsychotics. According to one study, these practice directives have achieved some success when 10 -year prescribing trends are examined, with SRI use increasing from 50 to $59 \%$ in veterans diagnosed with PTSD, and benzodiazepine use diminishing by $7 \%$, although this reduction was offset by $9 \%$ increases in the use of zolpidem, a somewhat similar drug for sleep, and low-dose quetiapine. ${ }^{8}$ But if SRI drugs have been shown repeatedly to be no better than placebo in treating this population, yet quetiapine was effective in one high-quality trial, ${ }^{9}$ what sort of triumph does this represent? Harpaz-Rotem and associates note that antidepressants are more likely to be given for severe numbing and offered sooner after the diagnosis is made, tallying with the overlap of certain numbing symptoms and depression.

\section{Benzodiazepines and anxiolytics}

It is understandable that benzodiazepine and kindred drugs are more likely to be prescribed for severe sleep disturbance and intrusive symptoms such as distressing dreams and nightmares, which often respond poorly to SRI, but we lack data that speak to their effect. A rather meagre literature indicates that benzodiazepines are ineffective as monotherapy for the core features of PTSD, but it is possible that other GABA-A modulators, not so different 
from benzodiazepines, are efficacious. ${ }^{10}$ The GABA-acting drug, tiagabine, is however not effective in civilian PTSD. ${ }^{11}$ Benzodiazepines and related drugs are mostly off patent, are not actively promoted by the pharmaceutical industry, have been poorly studied in PTSD, yet continue to be used, as found again by Harpaz-Rotem in $42 \%$ of medication takers, despite a generally unfriendly prescribing climate that discourages their use. What might be the reasons for their persistence? A rapid onset of action, generally favourable side-effect profile, 'clean-up' actions against certain PTSD symptoms that do not always respond to SRI and potential effect on comorbid anxiety might all be explanations. At the same time, it would be foolish to remain unconcerned about the finding by Harpaz-Rotem that benzodiazepine and other anxiolytics are preferred in patients with diagnoses of personality disorders and histories of drug-use disorder, given the possible risk of misuse and behavioural disinhibition. (We do not know the type of personality disorders in their sample - an important question.) Quite clearly, PTSD with high re-experiencing, dysphoric arousal and a history of personality aberration or drug abuse remains a management challenge, and the general exclusion of patients with these characteristics from randomised controlled trials means that we know relatively little about which treatments work and which treatments harm. Glimmers of light in this respect seem to suggest a poor effect of sertraline versus placebo ${ }^{12}$ and an inferior effect of paroxetine versus desipramine ${ }^{13}$ in PTSD with comorbid alcohol dependence. Tricyclics are understudied, and perhaps greater attention could be directed to low-dose tricyclics for sleep disturbance as an alternative to benzodiazepine and related anxiolytics. Benefits on sleep could be one reason why the use of quetiapine increased by $9 \%$.

\section{Antipsychotics}

Antipsychotics were prescribed to $20 \%$ of those who received a drug and were favoured in the presence of severe numbing and reexperiencing, a finding that should be seen in the light of the most recent placebo-controlled trial of quetiapine in 80 veterans, which failed to demonstrate benefit on numbing, even though the drug was effective overall at an effect size of $0.49,{ }^{9}$ which is one of the highest obtained in psychopharmacology trials of PTSD. ${ }^{14}$ Perhaps antipsychotics vary in their effect on the DSM-IV Cluster C symptoms, reflective of different receptor-binding profiles, although norquetiapine, a metabolite of quetiapine, is a noradrenergic reuptake inhibitor and would be expected to ameliorate those particular symptoms. Harpaz-Rotem et al found that clinicians favoured antipsychotics where comorbid psychosis or bipolar disorder was present, and few would be surprised by this finding. What is far less clear, however, is whether some antipsychotics, and at lower doses, may warrant greater use in those without psychosis or bipolarity, with proper monitoring of side-effects.

\section{Prazosin}

Widespread use of the alpha-adrenergic antagonist prazosin for PTSD came about from a series of small trials based on observations made by Dr Murray Raskind that the drug, when given for other reasons, lead to amelioration of PTSD-related nightmares. In the prazosin trials, many participants received study drug as add-on therapy, but randomisation did not account for concomitant treatment, which complicates interpretation of the results. Encouraged by a positive study in 67 active duty military personnel, ${ }^{15}$ the VHA undertook a multicentre trial in over 300 patients. A preliminary report of this study shows no difference between drug and placebo. ${ }^{16}$ So we are again confronted with the finding that a drug proven efficacious in various populations failed to work in a large cohort of US veterans. What are the implications of this negative study on VHA guidelines? Does the outcome reflect a treatment-refractory population, inadequacy of the drug or the complex stage production of a large multicentre trial that results in a dilution of the drug signal, relative to all the other activities and expectations accompanying the study? Or could it be that treatment produces other benefits that were not measured?

\section{Polypharmacy}

The complexity of PTSD, its comorbidity and persistence of symptoms after initial treatment all increase the likelihood of polypharmacy, which in recent years has been extensive in the VHA. In 2004, 2009 and 2012, the prescription rates of at least three psychotropics in PTSD were 39,51 and $55 \% .{ }^{17}$ Major guidelines ${ }^{18-22}$ do not address in detail the use of combined drugs in PTSD, which can be thought of in two different ways: (i) the administration of two or more drugs and (ii) the administration of a single drug with multiple effects. These scenarios can be exemplified in a hypothetical patient with severe nightmares, who by current guidelines is likely to receive in combination a serotoninspecific reuptake inhibitor, with its effect limited to serotonin reuptake, plus prazosin, with its main effect on the alpha-1 adrenergic receptor. Alternatively, a single drug could be used, such as a tertiary amine tricyclic drug or quetiapine, which have multiple effects on serotonin and noradrenaline reuptake; antagonism of the histamine, $5-\mathrm{HT}_{2}$ and alpha- 1 adrenergic receptors; and for some tricyclic antidepressants, inhibition of monoamine oxidase. $^{5}$ With the second approach, some drug effects can be recruited differentially depending on the dose, for example, the powerful yet selective antihistamine effect of doxepin at an extremely low dose. What are the relative advantages and disadvantages of each course? Are 'dirty' drugs better for PTSD than 'clean' ones? When is it appropriate to introduce additional drugs? Harpaz-Rotem and colleagues rightly draw our attention to clinician choices of deciding which drug, when and for whom, and their use of dimensional symptom clusters is a promising approach which requires further elaboration in broadly defined PTSD populations and reminds us that pattern recognition is an inherent part of medical practice. At the least, it suggests that guidelines should be interpreted in context of the different presentations that characterise PTSD and that one drug may not fit all. Treatment algorithms can be a further useful guide to clinical practice beyond the first stage of drug therapy in PTSD, although existing algorithms, such as the web-based International Psychopharmacology Algorithm Project (IPAP) for PTSD, ${ }^{23}$ are now over a decade old and need updating to incorporate new evidence.

\section{Conclusions and future expectations}

Medication was more likely to be prescribed to those with severe PTSD symptoms and to those receiving psychotherapy. The challenges of treating PTSD within the VHA have been previously shown by two reports showing lack of general improvement in patients who went through specialised in-patient treatment programmes, as well as all comers. ${ }^{17,24}$ In the former category, scores on the PCL changed minimally from 65.9 pre-treatment to 60.2 after intensive multi-modality treatment. It remains unclear whether the treatment patterns found in the VHA as reported by Harpaz-Rotem and colleagues resemble delivery of medication in the civilian sector or in other large healthcare systems, such as in the United Kingdom, which formulate their own treatment policies and guidelines. 
The results obtained from applying PCL-derived symptom clusters suggest a need to better understand how VHA system physicians treat patients with high anxious arousal, that is, with startle and hypervigilance, since no drug class was preferred and anxiolytics were less likely to be given. Successful treatment of persisting startle can be difficult. The same can be said for avoidance symptoms. Based on the established anti-phobic effects of antidepressants and some benzodiazepines, these two drug classes may be useful for PTSD-related avoidance.

\section{Treatment advances: what can we expect?}

Most would agree that PTSD, as with nearly all psychiatric disorders, can profit from the development of new treatments, but what does this well-worn mantra mean in practice? What are we to expect? In order of probability, it is likely to mean the following. First, a new treatment could produce the same rate of success as existing ones, but without any identification of best responders: it then becomes a 'me-too' drug. Second, a new drug might produce the same rate of success as existing drugs, but perform best in a subgroup of patients, for example, men versus women, PTSD with or without alcohol-use disorder, PTSD from military trauma versus other trauma, PTSD refractory to many treatments versus other groups: it then becomes a 'niche' or 'positioned' drug. Third, a new treatment may result in an incrementally greater rate of response or remission in all cases, for example, a $5-10 \%$ greater rate of success: it thus becomes an overall more effective drug and, assuming good safety, could become a game changer. Fourth, drug therapy might enhance or accelerate the benefit of psychotherapy, for example, the use of D-cycloserine or 3,4-methylenedioxymethamphetamine (MDMA). Lastly, least likely but most hoped for is a drug which represents a true breakthrough - a drug such as ketamine which works within hours or which produces almost complete removal of symptoms. The last two scenarios would compel the clinician to think of pharmacotherapy interactively, in conjunction with either psychotherapy or other longer-term medication. Any of these five developments would represent a welcome advance.

Jonathan Davidson, MB, BS, FRCPsych, Department of Psychiatry \& Behaviora Sciences, Duke University Medical Center, Durham, NC, USA

Correspondence: Jonathan Davidson, 3068 Baywood Drive, Seabrook Island, SC 29455, USA. Email: david011@mc.duke.edu

First received 9 Aug 2016, final revision 27 Oct 2016, accepted 31 oct 2016

\section{References}

1 Harpaz-Rotem I, Rosenheck R, Mohamed S, Pietrzak R, Hoff R. Initiation of pharmacotherapy for post-traumatic stress disorder among Iraq and Afghanistan veterans: a dimensional, symptom cluster approach. Br J Psych Open 2016; 2 286-93.

2 VA/DoD Clinical Practice Guideline: Management of Post-Traumatic Stress. Guideline Summary. US Department of Veterans Affairs and the Department of Defense, 2010

3 Martenyi $F$, Brown EB, Zhang $H$, Prakash A, Koke SC. Fluoxetine vs placebo in posttraumatic stress disorder. J Clin Psychiatry 2002; 63: 199-206.

4 Panahi Y, Moghaddam BR, Sahebkar A, Nazari MA, Beirhagdar F, Karami G, et al. A randomized, double-blind, placebo-controlled trial on the efficacy and tolerability of sertraline in Iranian veterans with post-traumatic stress disorder Psychological Med 2011; 41: 2159-66.
5 Davidson JRT. Vintage treatments for PTSD: a reconsideration of tricyclic drugs J Psychopharmacol 2015; 29: 264-9.

6 Yehuda R, Hoge CW. Treatment options for veterans with PTSD - Reply. JAMA Psychiatry 2016; 73: 758.

7 Steenkamp MM. Treatment options for veterans with PTSD. JAMA PSychiatry 2016; 73: 757-8. doi:10.1001/jamapsychiatry.2016.0573.

8 Bernardy NC, Lund BC, Alexander B, Friedman MJ. Prescribing trends in veterans with posttraumatic stress disorder. J Clin Psychiatry 2012; 73: 297-303.

9 Villareal G, Hamner MB, Canive JM, Robert S, Calais LA, Durklaski V, et al. Efficacy of quetiapine monotherapy in posttraumatic stress disorder: a randomized, placebo-controlled trial. Am J Psychiatry 15 July 2016 (doi:10.1176/appi.ajp.2016. 15070967)

10 Pollack MH, Hoge EA, Worthington JJ, Moshier SJ, Wechsler RS, Brandes M, et al. Eszopiclone for the treatment of posttraumatic stress disorder and associated insomnia: a randomized, placebo-controlled trial I Clin Psychiatry 2011: 72. 89-97.

11 Davidson JRT, Brady KT, Mellman TA, Stein MB, Pollack MH. The efficacy and tolerability of tiagabine in a population with post-traumatic stress disorder. J Clin Psychopharmacol 2007; 27: 85-8.

12 Brady KT, Sonne S, Anton R, Randall CN, Back SE, Simpson K. Sertraline in the treatment of co-occurring alcohol dependence and posttraumatic stress disorder. Alcohol Clin Exp Res 2005; 29: 395-401.

13 Petrakis IL, Ralevski E, Desai N Trevisan L, Gueorgieva R, Rounsaville B, et al. Noradrenergic vs. serotonergic antidepressant with or without naltrexone for veterans with PTSD and comorbid alcohol dependence. Neuropsychopharmacology 2012; 37: 996-1004.

14 Hoskins M, Pearce J, Bethell A, Dankova L, Barbui C, Tol WA, et al. Pharmacotherapy for post-traumatic stress disorder: systematic review and metaanalysis. Br J Psychiatry 2015; 206: 93-100.

15 Raskind MA, Peterson $\mathrm{K}$, Williams $\mathrm{T}$, Hoff DJ, Hart $\mathrm{K}$, Holmes $\mathrm{H}$, et al. A trial of prazosin for combat trauma PTSD with nightmares in active-duty soldiers returned from Iraq and Afghanistan. Am J Psychiatry 2013; 170: 1003-10.

16 Cooperative Studies Program \#563 - Prazosin and Combat Trauma PTSD (PACT). Study Results (https://clinicaltrials.gov/ct2/show/results/NCT00532493?sect= xba9870156\#Outcome5)

17 IOM (Institute of Medicine). Treatment for Posttraumatic Stress Disorder in Military and Veteran Populations: Final Assessment. National Academies Press, 2014.

18 Baldwin DS, Anderson IM, Nutt DJ, Allgulander C, Bandelow B, den Boer JA, et al. Evidence-based pharmacological treatment of anxiety disorders, post-traumatic stress disorder and obsessive-compulsive disorder: a revision of the 2006 guidelines from the British Association of Psychopharmacology. J Psychopharmacology 2014; 28: 403-39.

19 National Collaborating Centre for Mental Health. Post-traumatic Stress Disorder: the Management of PTSD in Adults and Children in Primary and Secondary Care. NICE Clinical Guidelines (CG26). NICE, 2005.

20 International Society for Traumatic Stress Studies (ISTSS). Effective Treatments for PTSD: Practice Guidelines for the International Society of Traumatic Stress Studies. Guilford Press, 2009.

21 World Health Organization (WHO). Guidelines for the Management of Conditions Specifically Related to Stress (www.who.int/mental_health/resources/ emergencies). WHO, 2013.

22 Bandelow B, Zohar J, Hollander E, Kasper S, Möller HJ, WFSBP Task Force on Treatment Guidelines for Anxiety, et al. World Federation of Societies of Biological Psychiatry (WFSBP) guidelines for the pharmacological treatment of anxiety, obsessive-compulsive and post-traumatic stress disorders - first revision. World J Biol Psychiatry 2008; 9: 248-312.

23 Davidson JRT, Bernik M, Connor KM, Friedman, MJ, Jobson K, Kim Y, et al. A new treatment algorithm for posttraumatic stress disorder. Psychiatr Ann 2005. 35 : 887-902.

24 Fontana A, Rosenheck R. Effectiveness and cost of the inpatient treatment of posttraumatic stress disorder: comparison of three models of treatment. Am J Psychiatry 1997; 154: 758-65. 